MATHEMATICS OF COMPUTATION

Volume 73, Number 245, Pages 297-315

S $0025-5718(03) 01565-5$

Article electronically published on June 19, 2003

\title{
THE BRUMER-STARK CONJECTURE IN SOME FAMILIES OF EXTENSIONS OF SPECIFIED DEGREE
}

\author{
CORNELIUS GREITHER, XAVIER-FRANÇOIS ROBLOT, AND BRETT A. TANGEDAL
}

\begin{abstract}
As a starting point, an important link is established between Brumer's conjecture and the Brumer-Stark conjecture which allows one to translate recent progress on the former into new results on the latter. For example, if $K / F$ is an abelian extension of relative degree $2 p, p$ an odd prime, we prove the $l$-part of the Brumer-Stark conjecture for all odd primes $l \neq p$ with $F$ belonging to a wide class of base fields. In the same setting, we study the 2-part and $p$-part of Brumer-Stark with no special restriction on $F$ and are left with only two well-defined specific classes of extensions that elude proof. Extensive computations were carried out within these two classes and a complete numerical proof of the Brumer-Stark conjecture was obtained in all cases.
\end{abstract}

\section{Overview AND RESUlts}

An important conjecture due to Brumer predicts that specific group ring elements constructed from the values of partial zeta-functions at $s=0$ annihilate the ideal class groups of certain number fields. Recent progress has been made on this conjecture ([Gr1], WWi]) and this will be used to obtain new results on the related conjecture of Brumer-Stark where progress thus far has been more restricted (see Section 1 of $[\mathrm{RT}]$ for the present status of the Brumer-Stark conjecture). The setting for these conjectures is the following: $K / F$ is a relative Galois extension, with $G=\operatorname{Gal}(K / F)$ abelian, $K$ totally complex, and $F$ totally real. Both conjectures predict that certain elements of $\mathbb{Z}[G]$ annihilate the ideal class group $\mathrm{Cl}_{K}$ of $K$.

Our results fall naturally into two parts. The first part (Sections 1-3) presents our theoretical results, which we now briefly state:

(1) (Probably well known) There is the following localization principle: The Brumer-Stark conjecture (BS) holds for $K / F$ if and only if, for all prime numbers $l$, an "l-primary analog" (BS) $l$ holds for $K / F$.

(2) (Semi-simple case) If $F$ is an abelian extension of $\mathbb{Q}$ with the $l$-part of $\operatorname{Gal}(F / \mathbb{Q})$ cyclic, $K$ is a $\mathrm{CM}$ field, and $l \neq 2$ is prime to the order of $G$, then $(\mathrm{BS})_{l}$ holds. For example, given this setup, if $G$ is abelian of order $2 p, p$ an odd prime, then it suffices to prove (BS) $)_{2}$ and $(\mathrm{BS})_{p}$ in order to establish (BS) for $K / F$. (The condition that $F$ be abelian over $\mathbb{Q}$ can be relaxed somewhat; see Proposition 1.3 for details.)

(3) If $G$ is abelian of order $2 p, p$ an odd prime, then $(\mathrm{BS})_{p}$ holds unless $K / F$ is of type $\sharp$ or $b$. Here $\sharp$ means that $K$ contains a primitive $p$-th root of unity $\zeta_{p}$ and

Received by the editor December 20, 2001.

2000 Mathematics Subject Classification. Primary 11R42; Secondary 11R29, 11R80, 11Y40.

Key words and phrases. Algebraic number fields, Brumer-Stark conjecture. 
$K / F$ is unramified outside the primes above $p$. The case $b$ is even more exceptional; details will be given later. We shall see that, for instance, $b$ does not happen if $p=3$ or if $p=5$ and $F$ is real quadratic.

(4) Suppose $F$ is real quadratic and $K / F$ is cyclic of degree 6 . Then (BS) holds in full, except perhaps in case $\sharp$. In particular, we prove $(B S)_{2}$ without exception in this setting.

The second part (Section 4) presents our computational work, where certain situations not covered in full by our theoretical results are studied, for example:

(5) In the setting of (4): The "first" 534 instances of case $\sharp$ satisfy the full Brumer-Stark conjecture as well. We will of course give details on what "first" means. For the moment let us just say: A list of cases was generated, with various cut-off criteria and elimination of uninteresting cases, for instance cases where the minus class number of $K$ happened not to be divisible by 3 , and consequently $(\mathrm{BS})_{3}$, and (BS), are known to hold for simple reasons. This list, with elimination and search limits, came to 534 items, and (BS) was successfully verified in every case.

(6) We also carried out extensive computations involving cyclic extensions $K / F$ of degree 6 over real cubic fields $F$. Both $\sharp$ and $b$ type extensions arise in this scenario and we generated lists of nontrivial cases for both types using cut-off and elimination criteria. The Brumer-Stark conjecture was successfully verified in all $114 \sharp$ cases and all $145 b$ cases considered.

We have one further result we would like to mention here: (BS) holds in full when $F$ is real quadratic and $K / F$ is cyclic of degree 4 . This was previously the situation involving the lowest degree fields over $\mathbb{Q}$ still unproved (see $[\mathbf{R T}]$ ). We are

planning to treat this situation and give further results in particular involving the 2-primary part $(\mathrm{BS})_{2}$ in a sequel to the present paper.

\section{Statement of (BS) And its primary parts; THE LINK WITH THE BRUMER CONJECTURE}

For the first part of this section we assume that $K / F$ is an abelian $G$-Galois extension with $F$ totally real and $K$ totally complex. (Towards the end of this section and for the complete remainder of the paper we will make the extra assumption that $K$ is a CM field.) For simplicity, we make the blanket assumption throughout the paper that the base field $F$ is not equal to $\mathbb{Q}$, since the conjecture is known to be true in that case based upon Stickelberger's classic theorem concerning the factorization of Gauss sums (see [Ta2], p. 109).

Given an extension $K / F$ as above, let $S(K / F)$ denote the set of all primes of $F$, finite and infinite, that ramify in $K$. With respect to a finite set $S$ of primes in $F$ containing $S(K / F)$, we define a partial zeta-function corresponding to each element $\sigma \in G$ in the usual way:

$$
\zeta_{S}(s, \sigma)=\sum_{\sigma_{\mathfrak{a}}=\sigma} \frac{1}{\mathrm{Na}^{s}}
$$

where the sum is over all integral ideals $\mathfrak{a}$ of $F$ relatively prime to the finite primes in $S$ and having the same Artin symbol $(K / F, \mathfrak{a})=\sigma_{\mathfrak{a}}=\sigma$. The infinite sum on the right side converges only for $\Re(s)>1$, but $\zeta_{S}(s, \sigma)$ has a meromorphic continuation to all of $\mathbb{C}$ with exactly one (simple) pole at $s=1$. In particular, $\zeta_{S}(s, \sigma)$ is analytic at $s=0$, and based upon the work of Klingen [K] and Siegel Si] we know that $\zeta_{S}(0, \sigma) \in \mathbb{Q}$, and therefore the group ring element (referred to 
as a "Brumer-Stickelberger element")

$$
\theta_{S}(K / F)=\sum_{\sigma \in G} \zeta_{S}(0, \sigma) \sigma^{-1}
$$

lies in $\mathbb{Q}[G]$. We generally take $S=S(K / F)$ and in that case we will usually omit $S$ from the notation and simply write $\theta(K / F)$. If $F, K$, and $S$ are all fixed in a given context, we will sometimes just use $\theta$ to denote $\theta_{S}(K / F)$.

Let $w=w_{K}$ denote the order of the group of roots of unity $\mu_{K}$ in $K$. A famous result, due independently to Deligne and Ribet [DR], Barsky [Ba], and CassouNoguès $\left[\mathrm{CN}\right.$, states that $w \theta_{S}(K / F) \in \mathbb{Z}[G]$ and more generally $\xi \theta_{S}(K / F) \in \mathbb{Z}[G]$ whenever $\xi$ is an annihilator in $\mathbb{Z}[G]$ of $\mu_{K}$. An element $x \in K^{*}$ will be called $w$-abelian if its $w$-th root generates an extension field of $K$ which is abelian over $F$ (not just over $K$ ). An anti-unit is an element $x \in K^{*}$ which has absolute value 1 at all archimedean places of $K$ (recall that by our assumptions all these places are complex). If $K$ happens to be CM, with complex conjugation $j \in G$, then $x$ is an anti-unit if and only if $x^{1+j}=1$. We may now state the

Brumer-Stark Conjecture: For every nonzero fractional ideal $\mathfrak{a}$ of $K$, the ideal $\mathfrak{a}^{w \theta_{S}(K / F)}$ is principal and has a generator $x$ which is an anti-unit and $w$-abelian.

The first part stating that $\mathfrak{a}^{w \theta_{S}(K / F)}$ is always principal (i.e., that $w \theta_{S}(K / F)$ annihilates the class group $\mathrm{Cl}_{K}$ ) is due to Brumer (he predicts something even stronger; see below). The generator $x$ in the conjecture, if it exists, is unique up to a root of unity in $K$, and the prediction that such an element in $K$ exists with the special properties above is due to Stark. The set of nonzero fractional ideals $\mathfrak{a}$ that satisfy the conjecture forms a group under multiplication which contains all principal ideals (see Ta1 for this and other basic properties). Thus one may speak of the subgroup in $\mathrm{Cl}_{K}$ made up by the classes $[\mathfrak{a}]$ satisfying the BS property.

The $(\mathrm{BS})_{l}$ conjecture states, for any prime number $l$ : For every nonzero fractional ideal $\mathfrak{a}$ whose class is in the $l$-primary part $\mathrm{Cl}_{K}\{l\}$ of $\mathrm{Cl}_{K}$, the ideal $\mathfrak{a}^{w \theta_{S}(K / F)}$ is principal and has a generator $x$ which is an anti-unit and $w_{l}$-abelian. Here $w_{l}=|M|$, where $M=\mu_{K}\{l\}$. The criterion that $x$ be $w_{l}$-abelian can be stated as follows (see pp. 83-85 of [Ta2]): Let $\left\{\sigma_{i}\right\}_{i \in I}$ be a system of generators of $G$. Since $M$ is cyclic, there exists a system of integers $\left\{n_{i}\right\}_{i \in I}$ such that $\zeta^{\sigma_{i}-n_{i}}=1$ for all $\zeta \in M, i \in I$. The element $x \in K^{*}$ is $w_{l}$-abelian if and only if there exists a system of elements $\left\{\alpha_{i}\right\}_{i \in I}$ in $K^{*}$ such that $x^{\sigma_{i}-n_{i}}=\alpha_{i}^{w_{l}}$ for all $i \in I$ and $\alpha_{i}^{\sigma_{j}-n_{j}}=\alpha_{j}^{\sigma_{i}-n_{i}}$ for all $i, j \in I$. A weakened version of this criterion, sufficient for our purposes, will be proved in the course of proving Proposition 1.2 below.

Remark. If $l$ is odd, we may omit the condition that the generator $x$ is an anti-unit, by the following argument: For a given archimedean place $v$ of $F$ let $j_{v}$ be the nontrivial element of the decomposition group $G_{v} \subset G$. By property 3) on page 24-02 of [Ta1, we have $\left(1+j_{v}\right) \theta=0$, and hence $\left(1-j_{v}\right) \theta=\left(2-\left(1+j_{v}\right)\right) \theta=2 \theta$. On letting $A=\prod_{v}\left(1-j_{v}\right)$ (the product over the archimedean places $v$ of $F$ ), we thus obtain $A \theta=2^{a} \theta$ with $a=[F: \mathbb{Q}]$. Since squaring is a bijection on $\mathrm{Cl}_{K}\{l\}$, we find $\mathfrak{b}$ with $\mathfrak{a}=(u) \mathfrak{b}^{\mathfrak{a}^{a}}$ for some $u \in K^{*}$, and hence $\mathfrak{a}^{w \theta}=\left(u^{w \theta}\right) \mathfrak{b}^{w \theta A}$. Thus, a generator $z$ for $\mathfrak{b}^{w \theta}$ which is $w_{l}$-abelian immediately gives a generator $x=u^{w \theta} z^{A}$ for $\mathfrak{a}^{w \theta}$ which is again $w_{l}$-abelian, and it is an anti-unit since $1+j_{v}$ annihilates both $\theta$ and $A$ for all archimedean places $v$ of $F$. 
Proposition 1.1. The Brumer-Stark conjecture holds for $K / F$ if and only if $(B S)_{l}$ holds for every prime $l$.

Proof. It is obvious that (BS) implies (BS) $)_{l}$ for all primes $l$. To prove the converse, we have to show that the subgroup $U$ of classes satisfying the BS property is the whole of $\mathrm{Cl}_{K}$. For this it suffices that $U$ contains the $l$-primary part of $\mathrm{Cl}_{K}$ for all $l$, so fix some $l$ and take some $[\mathfrak{a}] \in \mathrm{Cl}_{K}\{l\}$. Then (BS) $l$ gives almost what we want, the only problem being that the generator is only $w_{l}$-abelian, and we want it to be $w$-abelian. To mend this, write $w=w^{\prime} w_{l}$ with $w^{\prime}$ prime to $l$ and use a similar trick just as before: Exponentiation by $w^{\prime}$ is bijective on $\mathrm{Cl}_{K}\{l\}$. Thus we find $\mathfrak{b}$ and $u \in K^{*}$ with $\mathfrak{a}=(u) \mathfrak{b}^{w^{\prime}}$. Let $\mathfrak{b}^{w \theta}=(z)$ with $z$ a $w_{l}$-abelian antiunit. Then $\mathfrak{a}^{w \theta}=\left(u^{w \theta} z^{w^{\prime}}\right)$, and the latter generator is now again an anti-unit and $w$-abelian.

Our next goal is to state and prove a result which links the Brumer conjecture to the Brumer-Stark conjecture and to explain which parts of the conjectures (the "semi-simple cases") are already known in some generality. To this end, we recall the Brumer conjecture. We keep our hypotheses on $K, F, G$, and $S$. The Brumer conjecture (B) then affirms that $I \theta_{S}(K / F)$ annihilates the class group $\mathrm{Cl}_{K}$, where $I$ is the annihilator in $\mathbb{Z}[G]$ of $\mu_{K}$. Just like the Brumer-Stark conjecture, (B) is equivalent to the collection of all $(\mathrm{B})_{l}, l$ running over all prime numbers, where $(\mathrm{B})_{l}$ means that $I_{l} \theta_{S}(K / F)$ annihilates the $l$-primary part of $\mathrm{Cl}_{K}$, with $I_{l}$ the annihilator of $M:=\mu_{K}\{l\}$ in $\mathbb{Z}_{l}[G]$, and $\mathbb{Z}_{l}$ denotes the $l$-adic integers.

C. Popescu has informed us that he has also obtained an essential part of the following result:

Proposition 1.2. Fix an odd prime $l$ and assume that, in the above notation, the module $M$ is $G$-cohomologically trivial. Then $(B)_{l}$ holds if and only if $(B S)_{l}$ holds.

Proof. We need two little preparations. First we claim: Whenever $M$ is not zero, the $l$-part $G\{l\}$ of the (abelian) group $G$ must be cyclic. Reason: $G\{l\}$ must act faithfully on $M$, otherwise a nontrivial $l$-subgroup $H \subset G$ would act trivially on $M$, and the cohomology of $H$ with coefficients in $M$ would be nontrivial. This easily implies that $G\{l\}$ is cyclic, since $M$ is cyclic of $l$-power order.

Second, there is the following group-theoretical observation: Suppose $G$ is abelian with cyclic $l$-part and assume there is an extension of groups

$$
1 \rightarrow C \rightarrow \Gamma \rightarrow G \rightarrow 1
$$

with $C$ an abelian $l$-group. Then $\Gamma$ is abelian if and only if the action of $G$ on $C$ via conjugation is the trivial action. (This is left to the reader. The main point is that the extension splits if $G$ has no $l$-part.)

Now assume that $(\mathrm{B})_{l}$ holds. Let $w_{l}=|M|$; recall $w=w_{K}=\left|\mu_{K}\right|$ and write $w=w_{l} w^{\prime}$ with $w^{\prime}$ prime to $l$. Since $M$ is cyclic, we have a canonical isomorphism $M \cong \mathbb{Z}_{l}[G] / I_{l}$. The hypothesis on cohomological triviality yields that $I_{l}$ is again cohomologically trivial; now $I_{l}$ has no $\mathbb{Z}$-torsion, hence $I_{l}$ is $\mathbb{Z}_{l}[G]$-projective, hence free on one generator, let us say $I_{l}=(\xi)$. Since $I_{l}$ contains a suitable $l$-power, it is a routine matter to show that we may choose $\xi \in \mathbb{Z}[G]$. Since $w_{l} \in I_{l}$ and $\xi$ is a nonzero divisor, we have

$$
w_{l}=\xi \cdot \nu \quad \text { for a unique } \nu \in \mathbb{Z}_{l}[G] .
$$


Given an ideal $\mathfrak{a}$ of $K$, we have to find a generator $x$ of $\mathfrak{a}^{w_{l} w^{\prime} \theta}$ which is $w_{l}$-abelian. (By a previous remark, we do not have to worry whether or not $x$ is an anti-unit.) Let $l^{N}$ be a high power of $l$ (larger than $w_{l}$ times the $l$-class number of $K$ ), and let $\nu^{\prime} \in \mathbb{Z}[G]$ be congruent to $\nu$ modulo $l^{N}$. Thus, $\mathfrak{a}^{w_{l} w^{\prime} \theta}$ is the product of a very high $l$-power of an ideal with the ideal $\mathfrak{a}^{\xi \nu^{\prime} w^{\prime} \theta}$. By hypothesis, $\mathfrak{a}^{\xi w^{\prime} \theta}$ is principal, with generator $y$, say. What remains is to show that $x=y^{\nu^{\prime}}$ has the "abelian property": $L:=K(\rho)$ is abelian over $F$ with $\rho^{w_{l}}=x$. We show that $L$ is normal over $K$ : The element $\bar{x} \in K^{*} / K^{* w_{l}}$ is annihilated by $\xi$, hence by the whole of $I_{l}$. Thus, for $\sigma \in G$, we find some integer $a$ with $\sigma-a \in I_{l}$ (this is the cyclicity of $M$ over $\mathbb{Z}$ ), and $\sigma(\bar{x})$ is just the $a$-th power of $\bar{x}$. Thus, all conjugates of $L / K$ over $K$ coincide with $L$.

Now we show that $L$ is abelian over $F$. If $w_{l}=1$, there is nothing to show, so we may assume $w_{l}>1$, which implies, as mentioned above, that the $l$-part of $G$ is cyclic. By virtue of the second preparatory remark, it suffices to show that the action by conjugation of $G$ on $\operatorname{Gal}(L / K)$ is the trivial one. Let $\sigma$ be any element of $G$ and lift it to an automorphism $\tilde{\sigma}$ of $L$ over $F$. Then we just have to show that $\tilde{\sigma}$ commutes with every automorphism $\tau: \rho \mapsto \zeta \rho$ of $L / K(\zeta \in M)$. If $a \in \mathbb{Z}$ such that $\sigma-a \in I_{l}$, then as seen above, $\tilde{\sigma}(\rho)$ must have the form $s \rho^{a}$ with $s \in K$. We get

and

$$
\tilde{\sigma} \tau(\rho)=\sigma(\zeta) \tilde{\sigma}(\rho)=\sigma(\zeta) s \rho^{a}
$$

$$
\tau \tilde{\sigma}(\rho)=\tau\left(s \rho^{a}\right)=\zeta^{a} s \rho^{a} .
$$

This is the same result as in the previous equation since $\sigma(\zeta)=\zeta^{a}$; thus $\operatorname{Gal}(L / F)$ is indeed abelian.

Remark. This last part of the argument is standard and well known. It actually shows that the property " $x$ is $w_{l}$-abelian" is equivalent in the present setting to the statement that $\bar{x}$ is annihilated by $I_{l}$, since $I_{l}$ is generated by terms of the form $\sigma-a$.

We now prove the reverse implication, so assume $(\mathrm{BS})_{l}$. Let $J$ be the group of nonzero fractional ideals in $K$. This is a torsion-free $\mathbb{Z}$-module, containing the submodule $P$ of principal ideals. It is convenient to calculate with the $l$-adic completions $J_{l}$ and $P_{l}$. Let $[\mathfrak{b}] \in \mathrm{Cl}_{K}\{l\}$. Then $\mathfrak{b}^{w_{l} w^{\prime} \theta}=(x)$, and $x$ satisfies the abelian condition, that is: The class of $x$ modulo $w_{l}$-th powers is annihilated by $I_{l}$. Thus $x^{\xi}$ can be written as $y^{w_{l}}$. In $P_{l}$ we then have equations

$$
(x)^{\xi}=(y)^{w_{l}}=(y)^{\nu \xi}
$$

since $\xi$ divides $w_{l}$ in $\mathbb{Z}_{l}[G]$ and $P_{l}$ has no torsion, we may cancel $\xi$ and obtain $(x)=(y)^{\nu}$. Now $(x)$ is $\mathfrak{b}^{w_{l} w^{\prime} \theta}=\left(\mathfrak{b}^{\xi w^{\prime} \theta}\right)^{\nu}$. With the same argument we may cancel the exponent $\nu$ and find $\mathfrak{b}^{w^{\prime} \xi \theta}=(y)$ in $J_{l}$. Since $\left[\mathfrak{b}^{\xi \theta}\right] \in \mathrm{Cl}_{K}\{l\}$, we may conclude that $\mathfrak{b}^{\xi \theta}$ is principal. Since $\xi$ generates $I_{l}$, we have proved the $l$-part of the Brumer conjecture.

This proposition will be important in the proof of the next result.

We will continue to keep all of the assumptions made at the beginning of this section, but from now onward we impose the extra hypothesis that $K$ is a CM field. Thus, $K$ is a quadratic extension of a totally real subfield $K^{+}$. Necessarily, the 
totally real field $F$ lies in $K^{+}$. The nontrivial element $j$ of $\operatorname{Gal}\left(K / K^{+}\right)$coincides with all $j_{v}$ in earlier notation; it is "the" complex conjugation in $G=\operatorname{Gal}(K / F)$.

Proposition 1.3. If the relative degree $[K: F]$ is prime to $l$ (which still is an odd prime), then the l-part of the Brumer conjecture is true for $K / F$ under either of the following two conditions:

(a) $F / \mathbb{Q}$ is abelian and the l-part of $\operatorname{Gal}(F / \mathbb{Q})$ is cyclic, or

(b) $F$ is cubic over $\mathbb{Q}$, and $l^{2}$ does not divide the discriminant of $F$. (Remark: We do not strive for maximum generality here but rather wish to provide the results we need later in the paper.)

Proof. We use character decompositions: For every $l$-adic character $\chi$ of $G$, there is an exact functor $M \rightarrow M_{\chi}$ on $\mathbb{Z}_{l}[G]$-modules, defined by base change: $M_{\chi}=$ $M \otimes_{\mathbb{Z}_{l}[G]} \mathbb{Z}_{l}[\chi]$. The functor $M \rightarrow M_{\chi}$ is naturally isomorphic to the functor $M \rightarrow M^{\chi}$ in Wi]. This property, and the exactness, are due to the circumstance that $l$ does not divide the order of $G$. Every module $M$ decomposes as a direct sum of $M_{\chi}$, with $\chi$ ranging over the $\mathbb{Q}_{l}$-conjugacy classes of all characters of $G$. The $l$-part of the Brumer conjecture for $K / F$ is, in the present situation, equivalent to the conjunction of all the $\chi$-parts of this conjecture, in an obvious sense. Note that since $l \neq 2$ and $F \neq \mathbb{Q}$, the Brumer-Stickelberger element has zero plus part and it suffices to consider the Brumer conjecture on $A(K)$, which denotes the $l$-part of the minus class group of $K$. In particular we may restrict attention to odd characters in what follows.

Now we begin with case (a). Standard module theory over discrete valuation rings shows that the $\chi$-part of the $l$-part of the Brumer conjecture is a consequence of the following statement à la Leopoldt-Iwasawa:

$$
\left|A(K)_{\chi}\right| \text { divides }\left[\mathbb{Z}_{l}(\chi):(\chi(\theta(K / F)))\right] \cdot\left|\left(\mu_{K}\{l\}\right)_{\chi}\right|,
$$

for any odd character $\chi$ of $G$. This formula follows directly from the statement of Theorem 3 in [Wi]; note that the equality given there becomes a divisibility statement since there may be primes ramifying in $K / F$ but not in the conductor of $\chi$, which gives extra Euler factors. This theorem is stated and proved under the hypothesis that a certain set $S_{\chi, l}$ is empty. So if this set is empty, we are done. If it isn't empty, then by the very definition of $S_{\chi, l}$, the character $\chi$ must be inflated from a character $\chi_{0}$ of $F^{c l}\left(\zeta_{l}\right) / F$, where the superscript ${ }^{c l}$ means taking the normal closure over $\mathbb{Q}$. Of course we may omit this superscript here. Write $K_{0}$ for $F\left(\zeta_{l}\right)$. Then we have a canonical isomorphism $A(K)_{\chi} \cong A\left(K_{0}\right)_{\chi_{0}}$, due to the fact that $l$ does not divide $\left[K: K_{0}\right]$. On the other hand, $\chi(\theta(K / F))$ equals $\chi_{0}\left(\theta\left(K_{0} / F\right)\right)$ times a product of Euler factors, by standard properties of $L$-functions.

This means that we are left with proving the above divisibility statement under the extra hypothesis that $K=F\left(\zeta_{l}\right)$ and thus $K$ is absolutely abelian (we now write again $K$ for $K_{0}$ and $\chi$ for $\left.\chi_{0}\right)$. Again, this can be extracted from the literature: We will use a result of Solomon $\left[\mathrm{So}\right.$. Let $\Gamma=\operatorname{Gal}(K / \mathbb{Q})$ and $\Gamma^{\prime}$ the non-l-part of $\Gamma$. Then $G=\operatorname{Gal}(K / F)$ is a subgroup of $\Gamma^{\prime}$, and $A(K)_{\chi}$ is the direct sum of all $A(K)_{\eta}$ with $\eta$ running over all characters up to conjugacy of $\Gamma^{\prime}$ that restrict to $\chi$ on $G$. We use the formula for $A(K)_{\eta}$ at the top of page 6 of [Gr2], which is based on [So, and we take the product over all $\eta$ up to conjugacy. (The required condition that the $l$-part of $\Gamma$ is cyclic does hold, thanks to the corresponding condition we imposed on $F$, and also thanks to $K=F\left(\zeta_{l}\right)$.) This gives the following formula 
in which $\psi$ runs over all characters of the whole group $\Gamma$ whose restriction to $G$ is conjugate to $\chi$ :

$$
\left|A(K)_{\chi}\right|=\prod_{\eta \mid G \sim \chi} l^{\delta_{\eta}} \cdot \prod_{\psi \mid G \sim \chi}\left|\mathbf{B}_{1, \psi^{-1}}\right|_{l} .
$$

Here $|b|_{l}=l^{v_{l}(b)}$, where $v_{l}: \overline{\mathbb{Q}_{l}} \rightarrow \mathbb{Q}$ is the extension of the $l$-adic valuation, so $v_{l}(l)=1$. The quantity $\delta_{\eta}$ is defined as follows: It is 1 unless $\eta$ is the Teichmüller character, and in that case it equals $r$ with $l^{r}=\left|\mu_{K}\{l\}\right|$. Since $\mathbf{B}_{1, \psi^{-1}}=$ $-L\left(0, \psi^{-1}\right)$, and since $L$-functions are fully inductive, we get

$$
\prod_{\psi \mid G \sim \chi}\left|\mathbf{B}_{1, \psi^{-1}}\right|_{l}=\prod_{\chi 1}\left|L_{F}\left(0, \chi_{1}^{-1}\right)\right|_{l}=\left[\mathbb{Z}_{l}(\chi):\left(L_{F}\left(0, \chi^{-1}\right)\right)\right] .
$$

On the other hand, $\chi(\theta(K / F))$ is a multiple of $L_{F}\left(0, \chi^{-1}\right)$. Moreover it is easy to check that $\prod_{\eta \mid G \sim \chi} l^{\delta_{\eta}}$ is exactly the order of the $\chi$-part of $\mu_{K}\{l\}$. This concludes the verification of statement $(*)$.

It remains to handle case (b). We may assume $F$ is not abelian over $\mathbb{Q}$. Again we must show that for all characters $\chi$ of $G$ the set $S_{\chi, l}$ is empty, so suppose $\mathfrak{q} \in S_{\chi, l}$. Then we have by the definition in Wi]: $\mathfrak{q}$ divides $l ; \chi$ factors through $\operatorname{Gal}\left(F^{c l}\left(\zeta_{l}\right) / F\right) ; \chi(\mathfrak{q})=1$ and the order of $\chi(m$, say) is at least 3 . We show that such a setup cannot exist.

Indeed the condition that $l^{2}$ does not divide $d=\operatorname{disc}(F)$ gives that the ramification degree of $\mathfrak{q} \mid l$ is at most 2. Since $F^{c l} / F$ is composed from the quadratic extension $\mathbb{Q}(\sqrt{d}) / \mathbb{Q}, \chi$ actually factors through a faithful character of $\operatorname{Gal}(M F / F)$ with $M$ a cyclic degree $m$ subextension of $\mathbb{Q}\left(\sqrt{d}, \zeta_{l}\right)$. Since $m>2$, the ramification exponent of $l$ in $M$ is $m$, or possibly $m / 2$ if $m / 2$ is odd. Therefore all primes of $M F$ above $l$ have ramification degree at least 3 . On the other hand $\mathfrak{q}$ does not ramify in $M F / F$ since $\chi(\mathfrak{q})=1$. This is a contradiction.

Corollary 1.4. Assume $F$ is a field satisfying the conditions in either part (a) or (b) of Proposition 1.3. If $K / F$ is as above (K is a $C M$ field) with $[K: F]$ prime to $l$ (l an odd prime), then the l-part of the Brumer-Stark conjecture is true for $K / F$.

Proof. The module $M=\mu_{K}\{l\}$ is $G$-cohomologically trivial for the simple reason that its cardinality is coprime to the order of $G$; we may thus invoke Propositions 1.2 and 1.3

\section{The $p$-PRImary Part of (BS) IN CASE $[K: F]=2 p$}

We assume in this section that the abelian group $G=\operatorname{Gal}(K / F)$ is of order $2 p$, $p$ an odd prime number, with $F$ totally real and $K$ totally complex. The field $K$ is automatically CM since each $j_{v}$ in earlier notation is equal to the unique element $j$ of order 2 in the cyclic group $G$. In this setting, Corollary 1.4 gives a widely applicable result covering (BS $)_{l}$ with $l \neq p$ an odd prime. We study $(\mathrm{BS})_{p}$ in this section and $(\mathrm{BS})_{2}$ in Section 3. Some extra notation:

$E$ denotes the quadratic extension of $F$ sitting in $K$.

$H=\operatorname{Gal}(K / E)$, which we will identify with $\operatorname{Gal}\left(K^{+} / F\right)$; hence $H$ has order $p$.

$\sigma$ is a fixed generator of $H$.

$j \in G$ is complex conjugation.

Thus $G$ is generated by $j \sigma$. Just for this section we will use the following notation for a CM field $L: A_{L}=\mathrm{Cl}_{L}^{-}\{p\}$, the $p$-part of $\mathrm{Cl}_{L}^{-}$. Our approach is now to 
distinguish some cases and subcases, and in most of them we show how the conjecture can be deduced from the analytic class number formula with some algebraic tricks. Two classes of exceptional cases will remain where these arguments fail: The first one (called type $b$ ) is given by the condition that

$\zeta_{p} \notin K$ and primes splitting in $E / F$ do not ramify in $K / E$ and $K^{c l} \subset\left(K^{c l}\right)^{+}\left(\zeta_{p}\right)$, where the exponent ${ }^{c l}$ means: Taking normal closure over $\mathbb{Q}$; the second (type $\sharp$ ) is given by the condition

$\zeta_{p} \in K$ and $K / E$ is at most ramified at primes over $p$.

It will soon be discussed how frequently these exceptions occur.

We distinguish, first of all, the two major cases I and II: $\zeta_{p}$ is not, respectively is, contained in $E$. Note that $\zeta_{p} \in E$ if and only if $\zeta_{p} \in K$.

We further distinguish Cases I and II in two subcases: (a) some prime $\mathfrak{p}$ in $E$ which is split in $E / F$ ramifies in $K / E$, and subcase (b), the logical complement of (a). In Case II a simpler, equivalent, formulation of (b) will be given.

Proposition 2.1. $(B S)_{p}$ holds in Case I with the possible exception of $b$.

Proof. We first do the subcase (a). By Lemma 2.5] (below), the order of the subgroup $A_{K}^{H}$ fixed under $H$ in $A_{K}$ is strictly larger than the order of $A_{E}$. This will be important later.

In what follows, we always consider projections to minus parts. That is, $\theta(K / F)$ will be considered as an element of $\mathbb{Q}[H]$ (originally it is in $\mathbb{Q}[G]$ and $G=H \times\{j\}$; we send complex conjugation $j$ to -1$)$. In the same vein, $\theta(E / F)$ will be considered as an element of $\mathbb{Q}$. Now $\theta(K / F) \cdot N$ has an expression as $\epsilon \cdot N \cdot \theta(E / F)$ where $N=1+\sigma+\cdots+\sigma^{p-1}$ is the norm element attached to $H$ and $\epsilon$ is a product of Euler factors attached to the primes that ramify in $K$ but not in $E$. The hypothesis of case (a) is just chosen to ensure that (the minus projection of) $\epsilon$ is zero. In other words, $\theta(K / F)$ is annihilated by $N$, and we'll make the most of that: We write $\theta(K / F)=(\sigma-1) \alpha$ for some $\alpha \in \mathbb{Q}[H]$.

Now we consider the analytic class number formula which gives, thanks to $\zeta_{p} \notin E$ (overbar is the canonical map $\mathbb{Z}_{p}[H] \rightarrow \mathbb{Z}_{p}\left[\zeta_{p}\right]$ ):

$$
\begin{aligned}
\left|A_{K}\right| & =\left[\mathbb{Z}_{p}\left[\zeta_{p}\right]:(\bar{\theta}(K / F))\right]\left[\mathbb{Z}_{p}:(\theta(E / F))\right] \\
& =p \cdot\left[\mathbb{Z}_{p}\left[\zeta_{p}\right]:(\bar{\alpha})\right]\left[\mathbb{Z}_{p}:(\theta(E / F))\right] \\
\left|A_{E}\right| & =\left[\mathbb{Z}_{p}:(\theta(E / F))\right] .
\end{aligned}
$$

Some remarks concerning integrality: Both $\left|A_{E}\right|$ and $\left|A_{K}\right| /\left(p\left|A_{E}\right|\right)$ are integers (the latter since $\left|A_{E}\right|$ strictly divides $\left|A_{K}^{H}\right|$ and this divides $\left.\left|A_{K}\right|\right)$. Therefore $\theta(E / F) \in$ $\mathbb{Z}_{p}$ and $\bar{\alpha} \in \mathbb{Z}_{p}\left[\zeta_{p}\right]$, and this shows that $\alpha$ can also be taken in $\mathbb{Z}_{p}[H]$.

We can now show that $\theta(K / F)$ kills $A_{K}$ as follows: The module $(\sigma-1) A_{K}$ is, via multiplication by $\sigma-1$, an epimorphic image of $A_{K} / A_{K}^{H}$. This latter quotient has cardinal strictly less than $\left|A_{K}\right| /\left|A_{E}\right|$, so

$$
\left|(\sigma-1) A_{K}\right| \leq \frac{1}{p}\left|A_{K}\right| /\left|A_{E}\right| \leq\left[\mathbb{Z}_{p}\left[\zeta_{p}\right]:(\bar{\alpha})\right] .
$$

Now we argue over the discrete valuation $\operatorname{ring} R=\mathbb{Z}_{p}\left[\zeta_{p}\right]$. The $R$-module $(\sigma-1) A_{K}$ is annihilated by $\alpha$ as a consequence of the last equation; hence $A_{K}$ is annihilated by $\theta(K / F)=(\sigma-1) \alpha$. Since $K$ has no nontrivial $p$-th roots of unity, this annihilation statement already proves $(\mathrm{BS})_{p}$ by virtue of Proposition 1.2 . 
It remains to treat case (b). Again by Proposition 1.2 we just want to see that the $p$-part of the Brumer conjecture (minus Stark) holds for $K / F$. Here we rely on the paper Gr1]. Theorems 4.10 and 4.11 of that paper show the $p$-part of the Brumer conjecture under the condition that $K / F$ is "nice". Those theorems were not stated "prime by prime", and for this reason, one does not need the full "niceness" hypothesis for the $p$-part of the conjecture. An extension was called nice if a certain condition CP holds, and for all odd $l$, the module $\mu_{K}\{l\}$ is $G$-cohomologically trivial. This latter condition is clearly satisfied in our situation for $l=p$. The point is that we only need a part of condition CP which originally stated that no critical prime of $F$ splits from $K^{+}$to $K$. There are two kinds of critical primes: those $\mathfrak{p}$ that ramify in $K / F$ (first kind) and those $\mathfrak{q}$ which lie over a rational prime $q$ such that $K^{c l} \subset\left(K^{c l}\right)^{+}\left(\zeta_{q}\right)$ (second kind). It is easy to see from Gr1 that the $\mathfrak{q}$ with $q \neq p$ play no role for the proof of the $p$-part. One has only to look at $\S 4$ for this, since critical primes of the second kind never make an appearance in the earlier sections. Let's sum up. To ensure the validity of $(\mathrm{B})_{p}$ it is enough to check two things:

(CP1) no critical prime of the first kind splits from $K^{+}$to $K$;

(CP2) there exist no critical primes of the second kind that divide $p$.

The first condition is assured by the hypotheses of case (b). The second condition is equivalent to saying that we are not in the exceptional case b, by the very definition of exceptional primes of the second kind.

The subdivision of Case II $\left(\zeta_{p} \in E\right)$ is the same as in Case I: With subcase II(a) we mean that some prime $\mathfrak{p}$ in $F$ splits in $E$ and ramifies in $K$; subcase II(b) is then what is left. In contrast to Case I, we can make a much sharper statement concerning subcase II(b):

Remark. In subcase II(b), only primes over $p$ can ramify in $K / E$ (or equivalently, in $\left.K^{+} / F\right)$; in other words, subcase $\mathrm{II}(\mathrm{b})$ is identical with the exceptional case $\sharp$ defined above. Suppose the prime $\mathfrak{p}$ of $E$ does not divide $p$ and is ramified in $K$. Then the prime $\mathfrak{p}^{+}$below $\mathfrak{p}$ in $E^{+}=F$ ramifies in $K^{+}$, and the absolute norm of $\mathfrak{p}^{+}$is congruent to 1 modulo $p$ (see [FT], Theorem 28(ii), p. 145). But then, since $E=F\left(\zeta_{p}\right), \mathfrak{p}^{+}$must split in $E$ (see [Ja], Proposition 3.1, p. 131), which puts us in subcase II(a).

Proposition 2.2. $(B S)_{p}$ holds in subcase II(a); in other words, $(B S)_{p}$ holds in Case II with the possible exception of $\sharp$.

Proof. This is very similar to the first proposition. Lemma 2.5 gives that $A_{K}^{H}$ has order strictly larger than $A_{E}$. We now look again at the analytic class number formula. Let us first assume that $\zeta_{p^{2}}$ is not in $K$. Then

$$
\begin{aligned}
\left|A_{K}\right| & =p \cdot\left[\mathbb{Z}_{p}\left[\zeta_{p}\right]:(\bar{\theta}(K / F))\right]\left[\mathbb{Z}_{p}:(\theta(E / F))\right] \\
& =p^{2} \cdot\left[\mathbb{Z}_{p}\left[\zeta_{p}\right]:(\bar{\alpha})\right]\left[\mathbb{Z}_{p}:(\theta(E / F))\right] ; \\
\left|A_{E}\right| & =p \cdot\left[\mathbb{Z}_{p}:(\theta(E / F))\right] .
\end{aligned}
$$

Here $\alpha$ is defined exactly as in the proof of Proposition 2.1. The module ( $\sigma-$ 1) $A_{K}$ is an epimorphic image of $A_{K} / A_{K}^{H}$ which in turn has cardinality smaller than $\left|A_{K}\right| /\left|A_{E}\right|=p \cdot\left[\mathbb{Z}_{p}\left[\zeta_{p}\right]:(\bar{\alpha})\right]$. Therefore $(\sigma-1) A_{K}$ is annihilated by $\alpha$, so $A_{K}$ is annihilated by $\theta(K / F)$. The punch line is that we still have a margin: 
The conjecture $(\mathrm{BS})_{p}$ talks about the exponent $p \theta(K / F)$, so $(\mathrm{BS})_{p}$ is a direct consequence of the annihilation statement.

We still have to consider the case that $K$ contains a higher $p$-power root of unity. No new ideas come in. The final step just becomes a little technical. So assume $\left|\mu_{p^{\infty}}(K)\right|=p^{e}$ with $e \geq 2$. Then $\left|\mu_{p^{\infty}}(E)\right|$ is at least $p^{e-1}$. If it is $p^{e}$, the proof runs as before, so let us assume it is $p^{e-1}$. If one suitably replaces the factors immediately after the equality signs in the formulas for $\left|A_{K}\right|$ and $\left|A_{E}\right|$ displayed above, one ends up with the statement that the order of $(\sigma-1) A_{K}$ is strictly less than $p^{2}\left[\mathbb{Z}_{p}\left[\zeta_{p}\right]:(\bar{\alpha})\right]$ (so the factor $p$ has become $p^{2}$ ). This means that $(\sigma-1) A_{K}$ is annihilated by $(\sigma-1) \alpha$ and $A_{K}$ by $(\sigma-1) \theta$. We must show that this is strong enough to imply that $p^{e} \theta$ is a Brumer-Stark annihilator.

Now the action of $\sigma$ on $\mu_{p^{\infty}}(K)$ is exponentiation by $g$, with $g-1$ exactly divisible by $p^{e-1}$, and $x \in K$ is $p^{e}$-abelian if and only if $x^{\sigma-g}$ is a $p^{e}$-th power in $K$. For this it is sufficient that $x$ be of the form $z^{\gamma}, \gamma=\sigma^{p-1}+g \sigma^{p-2}+\cdots+g^{p-1}$, since $(\sigma-g) \gamma=1-g^{p}$, which is exactly divisible by $p^{e}$. It is thus sufficient to show that the equation $p^{e} \theta=\gamma \alpha(\sigma-1) \theta$ has a solution $\alpha$ in $\mathbb{Z}_{p}[G]$, for then every ideal $\mathfrak{a}^{p^{e} \theta}$ has a generator which is a $\gamma$-th power. Since $\theta$ is killed by the norm element, we may just solve the equation with $\zeta_{p}$ substituted for $\sigma$, thus: $p^{e}=\left(1-g^{p}\right) \cdot\left(\zeta_{p}-g\right)^{-1} \cdot \alpha \cdot\left(\zeta_{p}-1\right)$. This equation is indeed solvable, since $1-g^{p}$ is exactly divisible by $p^{e}$ and since $\zeta_{p}-g$ is associated to $\zeta_{p}-1$.

We now discuss the "range" of the exceptional cases. If $F / \mathbb{Q}$ is normal and $E / \mathbb{Q}$ is unramified over $p$, then $b$ is impossible. In case $\sharp$ we must have $E=F\left(\zeta_{p}\right)$; in particular, since $E / F$ is quadratic, the absolute degree of $F$ must be at least $(p-1) / 2$. If $F$ is real quadratic and $p>5$, case $\sharp$ is therefore ruled out; if $F$ is real quadratic and $p=5$, case $\sharp$ is only possible if $F=\mathbb{Q}(\sqrt{5})$ and $E=\mathbb{Q}\left(\zeta_{5}\right)$. With a view towards calculations, we also show:

Lemma 2.3. For $p=3$ and $p=5$, case $b$ does not happen if $F$ is real quadratic.

Proof. Under the assumption $\zeta_{p} \notin E$, we prove that the inclusion $K^{c l} \subset\left(K^{c l}\right)^{+}\left(\zeta_{p}\right)$ cannot hold. Indeed, $K^{c l}$ is the composite $M M^{\prime}$, with $M$ quadratic or biquadratic over $F$ and with $M^{\prime}$ abelian of degree $p$ or $p^{2}$ over $F$. If the inclusion were true, we could pass to the maximal fields of exponent 2 over $F$, which would give

$$
M \subset M^{+}\left(\zeta_{p}\right) .
$$

Note that $\zeta_{p}$ has 2-power degree over $\mathbb{Q}$ and over $F$. The above inclusion would have to be an equality since $M$ is not real. Therefore $\zeta_{p}$ would lie in $M$. We show that this implies $\zeta_{p} \in E$ which finishes the proof. Indeed, $\zeta_{p}$ and $F$ generate an abelian extension of $\mathbb{Q}$. If $E$ is normal over $\mathbb{Q}$, then $E=M$ and we are done. If not, $M$ is dihedral of degree 8 over $\mathbb{Q}$ and $M=E E^{\prime}$ where $E^{\prime} / F$ is the unique conjugate of $E / F$ over $F$. Then the maximal subfield of $M$ abelian over $\mathbb{Q}$ is $E^{\prime \prime}$, the third relatively quadratic subfield of the biquadratic extension $E E^{\prime} / F$, and $\zeta_{p}$ must be in $E^{\prime \prime}$. On the other hand, $E / F$ and $E^{\prime} / F$ are both totally complex, so $E^{\prime \prime}$ would have to be totally real, a contradiction.

From Propositions 2.1 and 2.2 and Lemma 2.3 we obtain the following, which we point out as a corollary for later reference: 
Corollary 2.4. Let $K / F$ be a relative abelian extension with $K$ totally complex, $F$ totally real, and $[K: F]=2 p, p$ an odd prime. Then $(B S)_{p}$ is proved in general outside of cases $\sharp$ and $b$. If $F$ is real quadratic and $p=3$ or 5 , then $(B S)_{p}$ is proved except in case $\sharp$.

It remains to state and prove the lemma used in the proof of Proposition 2.1 Until the end of this section, we consider a cyclic Galois extension $K / E$ of $\mathrm{CM}$ fields of relative degree $p$ an odd prime and Galois group $H$. One defines the total ramification index $e(K / E)$ as the product over all ramification indices $e_{\mathfrak{p}}$ with $\mathfrak{p}$ running over the primes of $E$ that ramify in $K$. There is also the index $e^{-}(K / E)$ defined as $e(K / E) / e\left(K^{+} / E^{+}\right)$. The index $e^{-}(K / E)$ is equal to $p^{t}$ with $t$ the number of primes of $E^{+}$that split in $E$ and ramify in $K$. We recall that $A_{K}$ means the $p$-part of the minus part of $\mathrm{Cl}_{K}$.

Lemma 2.5. Suppose $H$ is cyclic of odd prime order $p$. Then

$$
\left|A_{K}^{H}\right|=\left|A_{E}\right| \cdot e^{-}(K / E) .
$$

Proof. From Lemma 4.1 on page 307 of $\mathrm{La}$ ] we have the formula

$$
\left|\mathrm{Cl}_{K}^{H}\right|=\frac{\left|\mathrm{Cl}_{E}\right| e(K / E)}{[K: E]\left[\mathcal{O}_{E}{ }^{*}: N_{K / E} K^{*} \cap \mathcal{O}_{E}{ }^{*}\right]} .
$$

An analogous formula holds with $K / E$ replaced by $K^{+} / E^{+}$. We now divide the former by the latter and extract the maximal $p$-power. On the left hand, we do end up with $\left|A_{K}^{H}\right|$. On the right hand, the quotient of $\left|\mathrm{Cl}_{E}\right|$ by $\left|\mathrm{Cl}_{E^{+}}\right|$contributes $\left|A_{E}\right|$. The quotient of the $e$-terms gives the $e^{-}$term. The term $[K: E]$ simply cancels against $\left[K^{+}: E^{+}\right]$, and what is most important, the quotient of the two norm indices gives

$$
\left[\mu_{E}: N_{K / E} K^{*} \cap \mu_{E}\right]
$$

by the theory of units in CM fields: The minus part of the multiplicative group of units is the group of roots of unity, and we may decompose into plus and minus parts with no worries since we are only interested in $p$-primary torsion.

We have to show that the above norm index is 1 . Indeed, let $\eta \in \mu_{E}$. We have to show it is in $N_{K / E} K^{*}$. By the Hasse norm theorem, this reduces to showing that $\eta$ is locally a norm in $K / E$. Now $K / E$ is induced from the cyclic extension $K^{+} / E^{+}$ by composing with $E$. Thus, locally, $\eta$ is a norm from $K$ if and only if $N_{E / E^{+}} \eta$ is a norm from $K^{+}$. But luckily the latter norm $N_{E / E^{+}} \eta$ is 1 since $\operatorname{Gal}\left(E / E^{+}\right)$is generated by complex conjugation.

\section{Completing the Proof for SeXtic extensions}

We suppose now that $F$ is totally real and $K$ is an abelian sextic CM extension of $F$, with Galois group $G$. From Proposition 1.1 and Corollary 1.4 we know that if $F$ is a real quadratic field, then (BS) holds for $K / F$ if and only if the local counterparts $(\mathrm{BS})_{l}$ hold for $l=2$ and $l=3$. Moreover, the case $l=3$ was proved in the preceding section (Corollary 2.4) for $F$ real quadratic, with the exception of one particular class of cases, called $\sharp$. The point now is that we can prove the case $l=2$ without exception, even over an arbitrary totally real field $F$. Actually the proof is quite similar in spirit to Sands' Sa1] treatment of the relative quadratic case, adorned with some slight algebraic complications. We do not push for maximum generality here: Certainly other abelian extensions could be treated as well. 
Let $G^{\prime}$ be the subgroup of order 3 of $G$ and let $K_{0}$ be the relatively quadratic subfield of $K / F$. (This was $E$ in the last section.) The group ring $\mathbb{Z}_{2}[G]$ contains two orthogonal idempotents $e=N_{G^{\prime}}$ and $e^{\prime}=1-e$; we have $e \mathbb{Z}_{2}[G] \cong \mathbb{Z}_{2}[j]$ and $e^{\prime} \mathbb{Z}_{2}[G] \cong \mathbb{Z}_{2}\left[\zeta_{3}\right][j]$. All $\mathbb{Z}_{2}[G]$-modules $M$ decompose as $M=e M \bigoplus e^{\prime} M$. Actually $e M$ is the part of $M$ belonging to the trivial character of $G^{\prime}$, and $e^{\prime} M$ belongs to either of the two nontrivial characters. Let $A$ (resp. $A_{0}$ ) be the 2-part of the class group of $K$ (of $K_{0}$, respectively); let $B$ (resp. $B_{0}$ ) be the factor group of $A\left(A_{0}\right)$ modulo the group of ambiguous ideals in the extension $K / K^{+}$(resp. $K_{0} / F$ ).

Since $\theta(K / F)$ maps to a multiple of $\theta\left(K_{0} / F\right)$, and since $(\mathrm{BS})_{2}$ holds for the relative quadratic extension $K_{0} / F$ by Sa1, we know right away that $A_{0}=e A$ (the group of ideal classes coming from $K_{0}$ ) is contained in the subgroup of $A$ of classes satisfying the $(\mathrm{BS})_{2}$ property. Thus we need only show the same thing for $e^{\prime} A$. This takes some preparation.

Let $d$ denote the number of finite primes of $K^{+}$that ramify in $K$, and let $d_{0}$ be defined accordingly for $K_{0} / F$. Then the analytic class number formula yields (cf. Sa1], Proposition 3.2):

$$
w_{K} \theta_{S\left(K / K^{+}\right)}\left(K / K^{+}\right) \sim 2^{\left[K^{+}: \mathbb{Q}\right]+d-2}|B|(1-j),
$$

where $\sim$ means that the two quantities differ by a unit factor in $\mathbb{Z}_{2}$; and a similar formula results on replacing $K$ by $K_{0}, K^{+}$by $F, B$ by $B_{0}$, and $d$ by $d_{0}$. (Bibliographical note: The factor $w_{K}$ is erroneously missing in the quoted proposition; however, it is present, as it should be, at the beginning of the proof of Proposition 5.21 ) of the same paper.) Let $\xi$ be the nontrivial character on $\{1, j\}$, and let $\chi_{0}, \chi_{1}, \chi_{2}$ be the three odd characters of $G$, numbered so that $\chi_{0}$ is trivial on $G^{\prime}$. We recall our notational convention that $\theta(K / F)=\theta_{S(K / F)}(K / F)$, with $S(K / F)$ precisely the set of places that ramify in $K / F$. We need a lemma:

Lemma 3.1. $\xi\left(\theta\left(K / K^{+}\right)\right)=\xi\left(\theta\left(K_{0} / F\right)\right) \cdot \chi_{1}(\theta(K / F)) \cdot \chi_{2}(\theta(K / F))$.

Proof. From well known inductive properties of $L$-functions we have

$$
L_{S}\left(K / K^{+}, \xi, s\right)=L_{S}\left(K / F, \chi_{0}, s\right) L_{S}\left(K / F, \chi_{1}, s\right) L_{S}\left(K / F, \chi_{2}, s\right)
$$

with $S=S(K / F)$ throughout. The first factor on the right is $L_{S}\left(K_{0} / F, \xi, s\right)$ and may be expressed as $L_{S\left(K_{0} / F\right)}\left(K_{0} / F, \xi, s\right)$ times $U$, with $U=\prod\left(1-\xi(\mathfrak{p}) N \mathfrak{p}^{-s}\right)$ and $\mathfrak{p}$ running over the primes of $F$ that ramify in $K$ but not in $K_{0}$. The expression on the left of the $=\operatorname{sign}$ is $L_{S\left(K / K^{+}\right)}\left(K / K^{+}, \xi, s\right)$ times $V$ with $V=\prod\left(1-\xi(\mathfrak{P}) N \mathfrak{P}^{-s}\right)$ and $\mathfrak{P}$ running over the primes of $K^{+}$that are unramified in $K$ but ramified over $F$. One sees easily that $U=V$, which gives, by cancellation:

$$
L_{S\left(K / K^{+}\right)}\left(K / K^{+}, \xi, s\right)=L_{S\left(K_{0} / F\right)}\left(K_{0} / F, \xi, s\right) L_{S}\left(K / F, \chi_{1}, s\right) L_{S}\left(K / F, \chi_{2}, s\right) .
$$

It now suffices to set $s=0$ and recall the construction of the Brumer-Stickelberger elements.

From Sands' formula (see above) we get

$$
|B|=w_{K} \xi\left(\theta\left(K / K^{+}\right)\right) \cdot 2^{-\left[K^{+}: \mathbb{Q}\right]-d+1}
$$

(note $1-j$ became a factor 2 upon applying $\xi$ ) and a similar formula for $\left|B_{0}\right|$. Since $\left|e^{\prime} B\right|=|B| /\left|B_{0}\right|$, we obtain by division, noting $w_{K}=w_{K_{0}}$ :

$$
\left|e^{\prime} B\right|=\chi_{1}(\theta(K / F)) \cdot \chi_{2}(\theta(K / F)) \cdot 2^{-\left[K^{+}: \mathbb{Q}\right]-d+[F: \mathbb{Q}]+d_{0}} .
$$


Now $e^{\prime} B$ is a module over the PID $R=\mathbb{Z}_{2}\left[\chi_{1}\right]=\mathbb{Z}_{2}\left[\zeta_{3}\right]$. We use $\alpha$ to denote $\chi_{1}(\theta(K / F)) \in R$. The exponent of 2 in the last formula is -4 or even more negative, so $\left|e^{\prime} B\right|$ divides the norm of $\alpha / 2^{2}$ from $R$ to $\mathbb{Z}_{2}$. This means that $\alpha / 2^{2}$ annihilates $e^{\prime} B$.

The step from this last statement to the desired statement that every class in $e^{\prime} A$ has the BS property is exactly the same as in Sa1, proof of (5.2) 1). (In our case, we have more factors of 2 than we actually need.)

We sum up what we have proved here:

Theorem 3.2. For $K / F$ sextic abelian with $F$ totally real and $K$ totally complex (automatically $C M),(B S)_{2}$ holds.

Applying Proposition 1.1] Corollaries 1.4 and [2.4, and Theorem [3.2] we finally obtain:

Theorem 3.3. Let $K / F$ be a sextic abelian extension with $K$ totally complex and $F$ either a real quadratic or a real cubic field. If $F$ is real quadratic, the Brumer-Stark conjecture holds in full unless $K / F$ falls under case $\sharp$. If $F$ is real cubic, abelian over $\mathbb{Q}$ or such that no square of a prime other than 2 or 3 divides its discriminant, then the Brumer-Stark conjecture holds in full unless $K / F$ falls under case $\sharp$ or case b. In all of these cases only $(B S)_{3}$ remains to be verified.

\section{Computations}

The Brumer-Stark conjecture has been proved in general when $G=\operatorname{Gal}(K / F)$ is cyclic of order 2 [Ta1] or bicyclic of order 4 [Sa1]. When $G$ is cyclic of order 4 and $F$ is real quadratic, Corollary 1.4 shows that only $(B S)_{2}$ requires verification. One reason we directed our efforts towards relative abelian sextic extensions was the intention of studying a situation where $(\mathrm{BS})_{3}$ is of paramount importance. Theorem 3.3 delineates for us exactly those extensions over real quadratic or real cubic base fields where $(\mathrm{BS})_{3}$ seems most difficult to prove. Our goal in this section is to give a complete numerical verification of the Brumer-Stark conjecture for a large number of examples within each of the three families of extensions where Theorem 3.3 falls short of a complete proof: $\sharp$ over real quadratic fields, $\sharp$ over real cubic fields, and $b$ over real cubic fields.

Our method of constructing the examples in this section is of interest in itself. In each case we start with a real quadratic or real cubic field $F$ satisfying the conditions in Theorem 3.3 We then need to construct a field $K^{+}$that is a cyclic cubic extension of $F$ (note that $K^{+}$is totally real as well) and has restricted ramification (for example, we only want ramification above the prime 3 with $\sharp$ extensions). A particularly attractive way to generate such extensions is offered by a related conjecture of Stark and we refer to $\mathrm{CO}$ or $[\mathrm{Ro}$. for a detailed explanation of this procedure. Even if we generate $K^{+}$based upon an unproved conjecture, an independent check can be made to ensure that a root of the irreducible cubic polynomial in $F[x]$ produced by Stark's conjecture truly does generate a cyclic cubic extension $K^{+} / F$ of the desired type. In the $\sharp$ cases, $K$ is obtained from $K^{+}$simply by adjoining $\zeta_{3}$. We need to be slightly more clever to obtain $b$ examples but $K^{+}$ is obtained there as well using Stark's related conjecture. Given an extension $K / F$ falling into one of our three categories, we still face the task of computing $\theta_{S}(K / F)$. In every case, $S$ was taken to be the minimal set $S(K / F)$ which is precisely the set of primes in $F$ that ramify in $K$. Throughout this section we simply write $\theta$ in place 
of $\theta_{S(K / F)}(K / F)$. A detailed explanation of how to compute $\theta$ over any totally real base field $F$ was given in [RT] and this method was used in all of our computations. Further details on our method of verifying the Brumer-Stark conjecture itself may be found in $[\mathrm{RT}]$ as well.

All computations were carried out using the PARI/GP package [PARI].

4.1. The real quadratic case. We generated 534 examples of type $\sharp$ over real quadratic fields using the search constraints listed below on $F$ and $K^{+}$. We have $K=K^{+}\left(\zeta_{3}\right)$ in each case:

(1) $F$ is real quadratic,

(2) $K^{+} / F$ is a cyclic cubic extension,

(3) $K^{+} / F$ is unramified outside 3 ,

(4) $d_{K^{+}} \leq 10^{12}$

(5) the minus class number of $K$ is divisible by 3 .

Note that if condition (5) does not hold, then $(B S)_{3}$ follows easily. Of the 534 extensions $K / F$ satisfying conditions (1)-(5), there are 91 where $K$ is abelian over $\mathbb{Q}$. When $K / \mathbb{Q}$ is abelian, Sands $[\mathrm{Sa2}$ and Hayes $[\mathrm{Ha}$ ] have proved the (BS) conjecture for $K / F$ with respect to the Brumer-Stickelberger element $\theta_{S H}=\theta \cdot \prod\left(1-\sigma_{\mathfrak{p}}^{-1}\right)$, with the product taken over all primes $\mathfrak{p}$ in $F$ ramified in $F / \mathbb{Q}$ but not ramified in $K / F$ (this product always includes at least one factor in each of the examples in our family). If all of the additional factors in $\theta_{S H}$ are 3 -adic units in the minus part of the group ring, then the result of Sands-Hayes applies to prove (BS) with respect to $\theta$ as well and this phenomenon occurred in 44 of our examples. For the remaining 490 examples, we computationally verified $(\mathrm{BS})_{3}$ to be true and therefore the full (BS) conjecture follows.

Proposition 4.1. The Brumer-Stark conjecture is true for all 534 extensions $K / F$ satisfying conditions (1)-(5) above.

We now give an example of such a verification. Let $F=\mathbb{Q}(\sqrt{69})$, let $K^{+}$be the ray class field of conductor 3 , and let $K=K^{+}\left(\zeta_{3}\right)$. The extension $K / F$ is a type $\sharp$ extension satisfying conditions (1)-(5) above. The field $K$ is generated over $\mathbb{Q}$ by an algebraic integer $\alpha$ satisfying

$$
\begin{aligned}
\alpha^{12}-6 \alpha^{11}+21 \alpha^{10}-44 \alpha^{9}+63 \alpha^{8}-72 \alpha^{7} & +103 \alpha^{6}-174 \alpha^{5}+51 \alpha^{4} \\
& +192 \alpha^{3}-63 \alpha^{2}-180 \alpha+144=0 .
\end{aligned}
$$

Let $\sigma$ be the generator of $\operatorname{Gal}(K / F)$ defined by

$$
\begin{aligned}
& \sigma(\alpha)=\frac{1}{39384}\left(805 \alpha^{11}-3995 \alpha^{10}+12649 \alpha^{9}-20944 \alpha^{8}+23354 \alpha^{7}\right. \\
& \left.-18508 \alpha^{6}+39459 \alpha^{5}-68889 \alpha^{4}-68817 \alpha^{3}+160020 \alpha^{2}+14454 \alpha-121896\right) .
\end{aligned}
$$

The element $\theta$ was computed to be equal to

$$
\theta=\frac{1}{3}\left(4+2 \sigma-2 \sigma^{2}-4 \sigma^{3}-2 \sigma^{4}+2 \sigma^{5}\right) .
$$

The class group of $K$ is of order 3 and $K^{+}$is a class number one field. The following ideal is a generator of $\mathrm{Cl}_{K}$ :

$$
\mathfrak{a}=2 \mathcal{O}_{K}+\left(1+\alpha^{4}+\alpha^{5}+\alpha^{8}\right) \mathcal{O}_{K}
$$


We computed $\mathfrak{a}^{3 \theta}$ and its class in the class group. We found that it is principal and generated by

$$
\begin{aligned}
z= & \frac{1}{256}\left(-289089+234063 \alpha-529186 \alpha^{2}-807811 \alpha^{3}-35091 \alpha^{4}+125033 \alpha^{5}\right. \\
& \left.-222916 \alpha^{6}-47864 \alpha^{7}+38987 \alpha^{8}-106532 \alpha^{9}+55649 \alpha^{10}-602512 \alpha^{11}\right) .
\end{aligned}
$$

We now look for a generator of $\mathfrak{a}^{3 \theta}$ that is also an anti-unit. It is not necessary to find a generator that is also an anti-unit in order to prove $(\mathrm{BS})_{3}$ by the remark preceding Proposition 1.1. But since this also gives a neat way to find a generator which is 3 -abelian (if $(\mathrm{BS})_{3}$ is true!), this seems to be the easiest way to proceed from a computational point of view. Here, it turns out that the generator $z$ is actually already an anti-unit (this was also true in almost all examples; this can be explained by the fact that PARI tries to return a generator as "small" as possible). Finally, $K\left(z^{1 / 3}\right)$ is abelian over $F$ if and only if $z^{N-\sigma}$ is a cube in $K$, where $N$ is an integer such that $\sigma\left(\zeta_{3}\right)=\zeta_{3}^{N}$; in this setting, it is easy to see that one can always take $N=-1$. We find

$$
\begin{aligned}
z^{-1-\sigma}= & {\left[\frac { 1 } { 6 3 0 1 4 4 } \left(-15453 \alpha^{11}+73560 \alpha^{10}-240326 \alpha^{9}+414447 \alpha^{8}\right.\right.} \\
& -568768 \alpha^{7}+595508 \alpha^{6}-1125963 \alpha^{5}+1585780 \alpha^{4}+586818 \alpha^{3} \\
& \left.\left.-1319271 \alpha^{2}-654420 \alpha+1880208\right)\right]^{3},
\end{aligned}
$$

and therefore $(\mathrm{BS})_{3}$ is true for this extension.

Listed below is a table of the structure of the minus 3-class groups occurring in the 490 examples we computationally verified. Each entry consists of two parts. The first part gives the invariant factor decomposition of an abelian group $A$ in the form $n_{1}, \ldots, n_{r}$ where $3 \mid n_{j}$ for all $j$ and $n_{j+1} \mid n_{j}$ for $1 \leq j<r$. The group $A$ has structure $C_{n_{1}} \times \cdots \times C_{n_{r}}$, where $C_{n}$ denotes the cyclic group of $n$ elements. The second part gives the number of minus 3 -class groups isomorphic to $A$.

\begin{tabular}{|c|c|}
\hline 3 & 258 \\
\hline 9 & 79 \\
\hline 27 & 10 \\
\hline 3,3 & 33 \\
\hline 9,3 & 76 \\
\hline
\end{tabular}

\begin{tabular}{|c|c|}
\hline 9,9 & 2 \\
\hline 27,9 & 5 \\
\hline $9,3,3$ & 20 \\
\hline $27,3,3$ & 4 \\
\hline $27,9,3$ & 3 \\
\hline
\end{tabular}

Remark. For $F$ real quadratic and $p=5$, case $\sharp$ is only possible if $F=\mathbb{Q}(\sqrt{5})$ and $E=\mathbb{Q}\left(\zeta_{5}\right)$ since $E / F$ must be a quadratic extension. To be in case $\sharp$ means that $K^{+} / F$ is cyclic of degree 5 and unramified outside 5 . A short calculation in local class field theory shows that $K^{+}$is then uniquely determined: It is the degree 10 field over $\mathbb{Q}$ of conductor 25 . The field $K=\mathbb{Q}\left(\zeta_{25}\right)$ has class number one and thus the full (BS) conjecture is true for $K / F$ by [Ta1].

4.2. The real cubic case. In this subsection we consider both type $\sharp$ and type $b$ extensions over real cubic fields. For $\sharp$ type extensions, we proceed similarly to the real quadratic case and consider all extensions $K / F$ with $K=K^{+}\left(\zeta_{3}\right)$ and $F$ and $K^{+}$satisfying the following constraints:

(1) $F$ is real cubic,

(2) $F / \mathbb{Q}$ is abelian or ( $q$ prime and $q^{2} \mid d_{F} \Rightarrow q=2$ or 3 ),

(3) $K^{+} / F$ is cyclic cubic, 
(4) $K^{+} / F$ is unramified outside 3 ,

(5) $d_{K^{+}} \leq 10^{15}$,

(6) the minus class number of $K$ is divisible by 3 .

Condition (2) is necessary to ensure that Theorem 3.3 applies and condition (4) ensures that $K / F$ is a type $\sharp$ extension. There are 114 extensions satisfying these conditions and 15 of these are such that $K$ is abelian over $\mathbb{Q}$. The result of SandsHayes ([Sa2], $\mathrm{Ha}$ ) did not apply in any of these abelian cases to prove the (BS) conjecture with respect to $\theta$ and therefore we computationally verified (BS) ${ }_{3}$ in all 114 examples using the same method as in the real quadratic case.

Proposition 4.2. The Brumer-Stark conjecture is true for all 114 extensions K/F satisfying conditions (1)-(6) above.

The following table gives the structure of the minus 3-class groups of these 114 fields:

\begin{tabular}{|c|c|}
\hline 3 & 47 \\
\hline 9 & 11 \\
\hline 27 & 1 \\
\hline 3,3 & 19 \\
\hline 9,3 & 22 \\
\hline
\end{tabular}

\begin{tabular}{|c|c|}
\hline 9,9 & 5 \\
\hline $3,3,3$ & 4 \\
\hline $9,3,3$ & 2 \\
\hline $9,9,3$ & 3 \\
\hline
\end{tabular}

In order to construct type $b$ extensions, we use the following tactic. Assume that the discriminant of $F$ has the form $d_{F}=3 d h^{2}$ with $d, h \geq 1, d$ square-free and $3 \nmid d$ ( $F$ is not abelian over $\mathbb{Q}$ since $d_{F}$ is not a square). Set $E=F(\sqrt{-d})$, and let $K^{+}$be a (totally real) cyclic cubic extension of $F$ such that all the primes ramified in $K^{+} / F$ are either ramified or inert in $E / F$. Now, let $K=K^{+} E=K^{+}(\sqrt{-d})$. It is clear that $\zeta_{3} \notin K$, but since $E^{c l}=F\left(\sqrt{-d}, \sqrt{d_{F}}\right)=F(\sqrt{-d}, \sqrt{-3}) \subset K^{c l}$, we see that $\zeta_{3} \in K^{c l}$ and actually $K^{c l}=\left(K^{c l}\right)^{+}\left(\zeta_{3}\right)$. Therefore $K / F$ is a type $b$ extension. Finally, in order to ensure that Theorem 3.3 applies, we must restrict the allowed values of $h$ to $1,2,3,6,9$, or $18\left(2^{3}\right.$ and $3^{5}$ are the highest powers of 2 and 3 , respectively, that can divide $d_{F}$ ). We thus consider all extensions $K / F$ with $F, K^{+}$, and $K$ satisfying the following constraints:

(1) $F$ is real cubic,

(2) $d_{F}=3 d h^{2}$ with $d$ square-free, $3 \nmid d$, and $h=1,2,3,6,9$, or 18 ,

(3) $K^{+} / F$ is cyclic cubic,

(4) $\mathfrak{p}$ ramified in $K^{+} / F \Rightarrow \mathfrak{p}$ ramified or inert in $F(\sqrt{-d}) / F$,

(5) $d_{K^{+}} \leq 10^{15}$

(6) the minus class number of $K=K^{+}(\sqrt{-d})$ is divisible by 3 .

There are 145 such extensions. For each of these we have computationally proved $(\mathrm{BS})_{3}$. However, we did not verify $(\mathrm{BS})_{3}$ directly. Indeed, the way these extensions are constructed implies that the discriminants of the fields $K$ are much larger than the ones we found for $\sharp$ type extensions over real cubic fields (the average discriminant of $K$ in our b examples is about $10^{40}$ as compared with about $10^{32}$ for the $\sharp$ examples). Finding generators of the ideals $\mathfrak{a}^{w_{K} \theta}$ in the $b$ case would require a tremendous amount of computer work. Fortunately, we can take advantage of the fact that $\zeta_{3} \notin K$ and deduce from Proposition 1.2 that $(\mathrm{BS})_{3}$ is equivalent to $(\mathrm{B})_{3}$ which is a lot easier to check computationally.

Proposition 4.3. The Brumer-Stark conjecture is true for all 145 extensions K/F satisfying conditions (1)-(6) above. 
We now give an example of how $(\mathrm{B})_{3}$ was checked in this setting. Let $F=\mathbb{Q}(\beta)$ with $\beta^{3}-15 \beta-11=0$ and $d_{F}=3^{3} \cdot 379$. If $K^{+}$is the ray class field modulo 3 and $K=K^{+}(\sqrt{-379})$, then $K / F$ is a type $b$ extension satisfying the conditions above. The field $K$ is generated over $\mathbb{Q}$ by an algebraic integer $\alpha$ satisfying

$$
\begin{aligned}
\alpha^{18}-18 \alpha^{16}-27 \alpha^{15}+135 \alpha^{14}+108 \alpha^{13}+345 \alpha^{12}+243 \alpha^{11}+7065 \alpha^{10} & \\
- & 26730 \alpha^{9}+47304 \alpha^{8}-183465 \alpha^{7}+212529 \alpha^{6}-337041 \alpha^{5} \\
& +1982187 \alpha^{4}-319113 \alpha^{3}+4234869 \alpha^{2}-1074978 \alpha+2138797=0 .
\end{aligned}
$$

Let $\sigma$ be the generator of $\operatorname{Gal}(K / F)$ defined by

$$
\begin{aligned}
\sigma(\alpha) & =\frac{1}{25642504251998015091363136823852538505350}\left(-25269652050923497929212771120026077 \alpha^{17}\right. \\
& +40102332900383807248126529375112654 \alpha^{16}+472143852594195097517708119595806967 \alpha^{15} \\
& \quad-40556171859845878461143042418164629 \alpha^{14}-4879316343979543590590886861288093147 \alpha^{13} \\
& +2143997949648729840779430938111638246 \alpha^{12}-472973717538113819727856636934455350 \alpha^{11} \\
& +12874314031468850703781916134606295925 \alpha^{10}-175034778726883761526482554920769518064 \alpha^{9} \\
& +937707788369275078182681363965190126961 \alpha^{8}-2147256501457017200394102724004023763715 \alpha^{7} \\
+ & 6063904950213330802965554608991729941733 \alpha^{6}-12246526239717823142768415853103938661601 \alpha^{5} \\
+ & 14923188674625299628566095708659108616482 \alpha^{4}-60032163251501835275377729140669965121226 \alpha^{3} \\
+ & 86713900497115826322509607741480020610254 \alpha^{2}-84338008294198391998255893276165939205806 \alpha
\end{aligned}
$$

We computed $\theta$ to be equal to

$$
\theta=60-60 \sigma-84 \sigma^{2}-60 \sigma^{3}+60 \sigma^{4}+84 \sigma^{5}=12\left(1-\sigma^{3}\right)\left(5-5 \sigma-7 \sigma^{2}\right) .
$$

The field $K^{+}$has class number one and the minus 3-class group of $K$ is isomorphic to $C_{27} \times C_{9} \times C_{3}$. Each cyclic component is generated, respectively, by the classes of the ideals $\mathfrak{a}_{1}, \mathfrak{a}_{2}$ and $\mathfrak{a}_{3}$ where

$$
\begin{gathered}
\mathfrak{a}_{1}=1346743 \mathcal{O}_{K}+\left(\alpha^{17}+113401 \alpha^{16}+164111 \alpha^{15}-6120 \alpha^{14}+60471 \alpha^{13}\right. \\
+192014 \alpha^{12}+588905 \alpha^{11}+301501 \alpha^{10}+227250 \alpha^{9}+176104 \alpha^{8} \\
-486356 \alpha^{7}+187668 \alpha^{6}+392157 \alpha^{5}+2273 \alpha^{4}-544623 \alpha^{3} \\
\left.+334792 \alpha^{2}+485257 \alpha+14022\right) \mathcal{O}_{K}, \\
\mathfrak{a}_{2}=21701161 \mathcal{O}_{K}+\left(\alpha^{17}+5967947 \alpha^{16}+6035814 \alpha^{15}+901785 \alpha^{14}\right. \\
+5050230 \alpha^{13}-2257687 \alpha^{12}+5599077 \alpha^{11}+10036919 \alpha^{10}+3808177 \alpha^{9} \\
+9283078 \alpha^{8}-797894 \alpha^{7}-5933662 \alpha^{6}+595599 \alpha^{5}-9968717 \alpha^{4}+4645261 \alpha^{3} \\
\left.-267451 \alpha^{2}-5650796 \alpha+2159654\right) \mathcal{O}_{K}, \\
\mathfrak{a}_{3}=31538539 \mathcal{O}_{K}+\left(\alpha^{17}+11072706 \alpha^{16}+7219250 \alpha^{15}-9719308 \alpha^{14}\right. \\
-14233721 \alpha^{13}-11309241 \alpha^{12}+9919126 \alpha^{11}+13593987 \alpha^{10}-1474838 \alpha^{9} \\
+3019847 \alpha^{8}+13482312 \alpha^{7}+2534813 \alpha^{6}+5113039 \alpha^{5}+4458431 \alpha^{4} \\
\left.-14063623 \alpha^{3}-864926 \alpha^{2}+8475998 \alpha+1373957\right) .
\end{gathered}
$$

We use the notation $(\mathfrak{b})$ to denote the class in the minus class group of the ideal $\mathfrak{b}$. Since $\zeta_{3} \notin K$, the annihilator of $\mu_{K}\{3\}$ is $I_{3}=\mathbb{Z}[G]$, so to verify the conjecture, it suffices to prove that $\mathfrak{a}_{i}^{\theta}$ is principal for $i=1,2,3$. We use the factorized form of $\theta$ to simplify the computations. Since $\mathfrak{a}_{3}$ is of order 3 , we have that $\mathfrak{a}_{3}^{12}$, hence $\mathfrak{a}_{3}^{\theta}$, is 
principal. The factor $1-\sigma^{3}$ won't contribute to the annihilation. Indeed, $\sigma^{3}$ acts as complex conjugation and $K^{+}$is a class number one field so that $\left(\mathfrak{b}^{\sigma^{3}}\right)=(\mathfrak{b})^{-1}$. Therefore $\left(\mathfrak{b}^{1-\sigma^{3}}\right)=(\mathfrak{b})^{2}$ and $1-\sigma^{3}$ induces an isomorphism of the minus 3-class group. We now look at the action of the factor $5-5 \sigma-7 \sigma^{2}$ :

$$
\begin{aligned}
\left(\mathfrak{a}_{1}^{5-5 \sigma-7 \sigma^{2}}\right) & =\left(\mathfrak{a}_{2}^{3} \cdot \mathfrak{a}_{3}^{2}\right), \\
\left(\mathfrak{a}_{2}^{5-5 \sigma-7 \sigma^{2}}\right) & =\left(\mathfrak{a}_{2}^{6}\right),
\end{aligned}
$$

so $\mathfrak{a}_{1}^{12\left(5-5 \sigma-7 \sigma^{2}\right)}$ and $\mathfrak{a}_{2}^{12\left(5-5 \sigma-7 \sigma^{2}\right)}$ are both principal, and hence so are $\mathfrak{a}_{1}^{\theta}$ and $\mathfrak{a}_{2}^{\theta}$. Thus $(\mathrm{B})_{3}$, and therefore $(\mathrm{BS})_{3}$, is true for the extension $K / F$.

The following table gives the structure of the minus 3-class groups of these 145 fields.

\begin{tabular}{|c|c|}
\hline 9 & 64 \\
\hline 27 & 15 \\
\hline 9,3 & 8 \\
\hline 9,9 & 9 \\
\hline 27,3 & 14 \\
\hline 27,9 & 5 \\
\hline
\end{tabular}

\begin{tabular}{|c|c|}
\hline 27,27 & 1 \\
\hline 81,3 & 3 \\
\hline 81,9 & 4 \\
\hline 243,3 & 1 \\
\hline $3,3,3$ & 5 \\
\hline $27,3,3$ & 4 \\
\hline $27,9,3$ & 5 \\
\hline
\end{tabular}

\begin{tabular}{|c|c|}
\hline $81,3,3$ & 1 \\
\hline $9,3,3,3$ & 1 \\
\hline $27,9,3,3$ & 1 \\
\hline $27,9,9,3$ & 2 \\
\hline $81,9,3,3$ & 1 \\
\hline $81,27,3,3$ & 1 \\
\hline
\end{tabular}

\section{REFERENCES}

[Ba] D. BARsky, Fonctions zêta $p$-adiques d'une classe de rayon des corps de nombres totalement réels. Groupe d'étude d'analyse ultramétrique 1977-78. Errata, idem 1978-79. MR 80g:12009

[CN] Pierrette Cassou-Noguès, Valeurs aux entiers négatifs des fonctions zêta et fonctions zêta p-adiques, Invent. Math. 51 (1979), 29-59. MR 80h:12009b

[Co] H. Cohen, Advanced Topics in Computational Number Theory, GTM 193, SpringerVerlag, New York, 2000. MR 2000k:11144

[DR] P. Deligne and K. Ribet, Values of abelian $L$-functions at negative integers over totally real fields, Invent. Math. 59 (1980), 227-286. MR 81m:12019

[FT] A. Fröhlich and M. TAYLOR, Algebraic Number Theory, Cambridge University Press, Cambridge, 1991. MR 94d:11078

[Gr1] C. Greither, Some cases of Brumer's conjecture for abelian CM extensions of totally real fields, Math. Zeitschrift 233 (2000), 515-534. MR 2001c:11124

[Gr2] An equivariant version of an index formula of Fröhlich and McCulloh, J. Number Th. 69 (1998), 1-15. MR 99c:11135

[Ha] David R. Hayes, Base change for the conjecture of Brumer-Stark, J. Reine Angew. Math. 497 (1998), 83-89. MR 99h:11131

[Ja] G. Janusz, Algebraic Number Fields, 2nd Ed., American Mathematical Society, Providence, 1996. MR 96j:11137

[Kl] H. Klingen, Über die Werte der Dedekindsche Zetafunktion, Math. Ann. 145 (1962), 265-272. MR 24:A3138.

[La] S. LAng, Cyclotomic Fields I and II, GTM 121, Springer-Verlag, New York, 1990. MR 91c:11001

[PARI] C. Batut, K. Belabas, D. Bernardi, H. Cohen, M. Olivier, The Computer Number Theory System PARI/GP, http://www.parigp-home.de/

[Ro] X. Rовцот, Stark's conjectures and Hilbert's twelfth problem, Experiment. Math. 9 (2000), 251-260. MR 2001g:11174

[RT] X. Roblot and B. TANGedal, Numerical verification of the Brumer-Stark conjecture, in: Algorithmic Number Theory, Proceedings of ANTS-IV Leiden (ed. W. Bosma), Lecture Notes in Computer Science 1838, Springer-Verlag, Berlin, (2000), 491-503. MR 2002e:11158 
[Sa1] J. SAnds, Galois groups of exponent two and the Brumer-Stark conjecture, J. Reine Angew. Math. 349 (1984), 129-135. MR 85i:11098

[Sa2] Abelian fields and the Brumer-Stark conjecture, Compos. Math. 53 (1984), 337346. MR 86c: 11102

[Si] C. Siegel, Über die Fourierschen Koeffizienten von Modulformen, Nachr. Akad. Wiss. Göttingen 3 (1970), 15-56. MR 44:2706

[So] D. Solomon, On the classgroups of imaginary abelian fields, Ann. Inst. Fourier, Grenoble 40 (1990), 467-492. MR 92a:11133

[Ta1] J. TATE, Brumer-Stark-Stickelberger, Séminaire de Théorie des Nombres Univ. Bordeaux I, Talence (1980-81) Exposé n 24. MR 83m:12018b

[Ta2] - Les Conjectures de Stark sur les Fonctions L d'Artin en $s=0$, Notes d'un cours à Orsay rédigées par D. Bernardi et N. Schappacher, Birkhäuser-Verlag, Boston, 1984. MR 86e: 11112

[Wi] A. WiLES, On a conjecture of Brumer, Ann. Math. 131 (1990), 555-565. MR 91i:11164

Institut FÜr theoretische Informatik und Mathematik, FAKUltät FÜr Informatik, Universität der Bundeswehr München, 85577 Neubiberg, F. R. Germany

E-mail address: greither@informatik.unibw-muenchen.de

Institut Girard Desargues, Université Claude Bernard (Lyon I), 69622 Villeurbanne, FRANCE

E-mail address: roblot@euler.univ-lyon1.fr

Department of Mathematics, College of Charleston, Charleston, South Carolina 29424-0001

E-mail address: tangedalb@cofc.edu 